\title{
Prognostic Stratification of Advanced Gastric Signet Ring Cell Carcinoma by Clinicopathological Factors and GALNTI 4 Genotype
}

\author{
Tsung-Hsing Chen ${ }^{1,2}$, Wey-Ran Lin ${ }^{1,5}{ }^{\bowtie}$, Chieh Lee 3 , Cheng-Tang Chiu ${ }^{1}$, Jun-Te Hsu ${ }^{4}$, Ta-Sen Yeh4, \\ Kwang-Huei Lin ${ }^{5,6,7}$, Puo-Hsien Le ${ }^{1}$, Chau-Ting Yeh ${ }^{1,5}$ \\ 1. Department of Gastroenterology and Hepatology, Linkou Chang Gung Memorial Hospital, Chang Gung University College of Medicine, Taoyuan, Taiwan \\ 2. Graduate Institute of Clinical Medical Sciences, Chang Gung University, Taoyuan, Taiwan \\ 3. Department of Industrial Engineering \& Management, Yuan Ze University College of Engineering, Chung-Li City, Taiwan \\ 4. Department of Surgery, Chang Gung Memorial Hospital, Linkou, Chang Gung University College of Medicine, Taoyuan, Taiwan \\ 5. Liver Research Center, Chang Gung Memorial Hospital, Linkou, Taoyuan, Taiwan \\ 6. Graduate Institute of Biomedical Sciences, Chang Gung University, Taoyuan, Taiwan \\ 7. Research Center for Chinese Herbal Medicine, College of Human Ecology, Chang Gung University of Science and Technology Taoyuan, Taiwan
}

$\triangle$ Corresponding authors: Chau-Ting Yeh, MD, PhD, Director, Liver Research Center, Linkou Chang Gung Memorial Hospital, 5, Fu-Shin St., Kuei-Shan District, Taoyuan, Taiwan. E-mail address: chautingy@gmail.com and Wey-Ran Lin, MD, Department of Gastroenterology and Hepatology, Linkou Chang Gung Memorial Hospital, 5, Fu-Shin St., Kuei-Shan District, Taoyuan, Taiwan. Email address: victor.wr.lin@gmail.com

(C) Ivyspring International Publisher. This is an open access article distributed under the terms of the Creative Commons Attribution (CC BY-NC) license (https://creativecommons.org/licenses/by-nc/4.0/). See http://ivyspring.com/terms for full terms and conditions.

Received: 2018.03.26; Accepted: 2018.07.27; Published: 2018.09.08

\begin{abstract}
Background: Gastric signet ring cell carcinoma (SRCC) is a histologic variant characterized by abundant intracytoplasmic mucin. Although it has been recognized that gastric adenocarcinoma harboring this feature has poorer prognosis, prognostic stratification within gastric SRCCs themselves has not been clearly defined. $\mathrm{N}$-acetylgalactosaminyltransferase 14 (GALNTI4) genotype has been associated to poorer treatment outcome in mucinous type colorectal cancer. Here we incorporated clinicopathological factors and GALNTI 4 genotype to stratify prognosis of advanced gastric SRCC.

Methods: Totally 347 gastric SRCC patients were retrospectively enrolled for GALNTI4 genotyping. Clinicopathological factors were included for prognosis stratification.

Results: Of the 347 patients, 341 underwent radical-intent gastrectomy and 6 received palliative gastrectomy. Kaplan-Meier analysis for overall survival indicated that Tumor-Node-Metastasis staging could only stratify the patients into three prognosis-distinguishable groups: group-1 (stage IA); group-2 (stage IB/IIA) and group-3 (the remaining Tumor-Node-Metastasis stages combined). Multivariate Cox-proportional hazard models for group-3 patients revealed GALNTI4 "TT" genotype $(P=0.0482)$. Tumor size $(P=0.0009)$, node status $(P$ $<0.0001)$, metastasis status $(P=0.0096)$, and perineural invasion $(P=0.037)$ independently associated with unfavorable OS. Exploratory subgroup analysis showed that GALNTI4"TT" genotype was associated with unfavorable OS in SRCCs with more aggressive phenotypes: node status $>0(P=0.0013)$, lymphatic invasion $(P$ $=0.021)$, vascular invasion $(P=0.0076)$ and perineural invasion $(P=0.0161)$. Accordingly, a scoring system was established capable of stratifying advanced gastric SRCC patients into three distinguishable prognostic subgroups.

Conclusions: Gastric SRCC could be stratified into different prognostic subgroups by combining clinicopathological factors and GALNTI 4 genotype.
\end{abstract}

Key words: GALNT14; signet ring cell; lymphatic invasion; vascular invasion; perineural invasion

\section{Introduction}

Gastric cancer is the third leading causes of cancer-related death in the world [1]. The incidence of gastric cancer is decreasing worldwide in recent decades but the incidence of signet ring cell carcinoma
(SRCC) increases progressively [2]. It is generally believed that gastric SRCC has a poorer prognosis compared with other types of gastric cancer [3-9]. SRCC is defined as a poorly cohesive carcinoma 
containing abundant intracellular mucin occupying more than $50 \%$ of the tumor volume, with formation of a crescent shaped nucleus in the peripheral area of cancer cells due to compression by mucin [5]. Although SRCC is considered a histological feature for poor prognosis in gastric cancer, the prognostic factors for gastric SRCC itself have not been defined. It is unclear whether the traditional Tumor-Node-Metastasis (TNM) staging system remains valid in stratifying prognosis for gastric SRCC. As the incidence of SRCC is increasing, it is imperative to establish an effective staging system for prognosis prediction within SRCC patients themselves.

$N$-acetylgalactosaminyltransferase 14 (GALNT14) encodes a Golgi protein, belonging to the polypeptide $\mathrm{N}$-acetylgalactosaminyltransferase protein family. The function of GALNTs is to transfer $\mathrm{N}$-acetyl-D-galactosamine to the serine/threonine residues of large proteins such as mucin. Mutation or knockdown of GALNT genes leads to developmental anomalies in mouse, fruit fly, and human [10]. GALNT14 has also been identified as a neuroblastoma predisposition gene [11]. To date, GALNT14-rs9679162 genotype has been demonstrated to be an effective outcome predictor in multiple gastrointestinal cancers including hepatocellular carcinoma, cholangiocarcinoma, esophageal cancer and colorectal cancer especially with mucinous histology [12-15]. Here, we hypothesized that GALNT14-rs9679162 genotype might also associate with the outcome of gastric SRCC.

\section{Patients and methods}

\section{Ethics statement}

The study protocol was approved by the Institutional Review Board of Chang Gung Memorial Hospital (104-8623B). Written informed consent was obtained for all participants, before surgical samples were deposited to Tissue Bank of Chang Gung Memorial Hospital.

\section{Patients}

Total 347 patients pathologically diagnosed as gastric SRCC at Chang Gung Memorial Hospital, Taoyuan, Taiwan, between 1996 and 2008, were enrolled. The Inclusion and Exclusion criteria were as followings. Inclusion criteria: patients who were diagnosed as gastric SRCC and received surgical treatment. Exclusion criteria: patients who were diagnosed as other types of gastric cancer (such as intestinal/diffuse type or mucinous gastric carcinoma).

Among these patients, 341 underwent radicalintent gastrectomy, while 6 stage IV patients received partial gastrectomy for symptomatic relief such as massive bleeding or obstruction. Subtotal or total gastrectomy was performed according to clinical conditions such as tumor size and location. The cancers were staged according to the seventh edition of the American Joint Committee on Cancer Tumor Node Metastasis classification [16]. All tissue samples were retrieved from Chang Gung Memorial Hospital tissue bank. The median follow-up time was 32.83 months, ranging from 0.4 to 254 months. Patients died after surgery during the same hospitalization periods were excluded for long-term survival analysis. Survival duration was calculated from the time of surgery to event (tumor recurrence or death) or the last follow-up, irrespective of the cause of death.

\section{GALNT14-rs9679162 genotyping}

Genotyping of GALNT14-rs9679162 was performed as previously described $[17,18]$.

\section{Statistical analysis}

Associations between the clinicopathological/ genetic factors and survivals were performed by use of Cox proportional hazard models. Factors that were considered of potential importance in the univariate analysis $(\mathrm{P}<0.15)$ were included in the multivariate analysis using forward mode stepwise selection. The rule-in level of significance was a $<0.15$ and rule-out level of significance was $a<0.10$. Statistical analyses were performed with SAS EG software for Windows, version 7.1 (SAS, Inc. USA).

Comparison of survivals between subgroups was performed using Kaplan-Meier analysis and evaluated by log-rank test. $\mathrm{P}<0.05$ was considered statistical significance.

\section{Results}

\section{Baseline demographics and clinicopathological data for all patients included}

The baseline demographics and clinicopathological features of patients who received potentially curative gastrectomy were summarized in Supplementary Table $\mathbf{1}$. They were $57.96 \pm 13.79$ years of age. No clear gender preference was present. Tumor staging was made according to the results of postoperative pathological examination. American Joint Committee on Cancer (AJCC), $7^{\text {th }}$ edition was applied. Of them, 6 patients were classified as stage IV and only partial gastrectomy for symptomatic relief was performed. More than half $(57.11 \%)$ of the gastric SRCCs were located in prepyloric area. GALNT14rs9679162 genotyping was performed using noncancerous gastric tissues. Of these patients, $23.34 \%, 59.65 \%$, and $17.00 \%$ were determined as "TT", "TG", and "GG" genotypes, respectively. Overall 
survival (OS) rate in 1, 2, 5, and 10 years was $77.81 \%$, $66.28 \%, 48.13 \%$, and $30.26 \%$, respectively.

\section{Clinicopathological factors associated with OS for all patients included}

The OS curves for all TNM stages were depicted in Fig. 1A. According to Kaplan-Meier analysis, these patients could only be divided into three prognosis-distinguishable groups (group-1: stage IA; group-2: stage IB/ IIA and group-3 other stages combined) (Fig. 1B). (The $\mathrm{P}$ values between group-1 vs 2; group-2 vs 3; and group-1 vs 3: 0.0499; <0.0001; $<0.0001)$. Notably, by use of Kaplan-Meier analysis, the TNM subgroups in group-3 patients (IIB, IIIA, IIIB, IIIC, and IV) could not be clearly separated in term of OS.

Univariate and multivariate Cox proportional hazard models were performed to understand the associations between clinicopathological factors and OS (Table 1). Univariate analysis identified tumor size, age, tumor $(\mathrm{T})$ status, node $(\mathrm{N})$ status, metastasis (M) status, lymphatic invasion, vascular invasion, perineural invasion, and TNM staging as associated factors (see Table 1 for each $P$ value).

Multivariate Cox proportional hazard analysis was performed using stepwise method (see Method). It was found that only tumor size $(\mathrm{P}=0.001), \mathrm{N}$ status $(\mathrm{P}<0.0001), \mathrm{M}$ status $(\mathrm{P}=0.0096)$, and perineural invasion $(\mathrm{P}=0.0339)$ remained in the equation. Intriguingly, the TNM stage was not included in the final model, suggesting that the TNM staging method was not an optimal prognosis classification system for gastric SRCC.
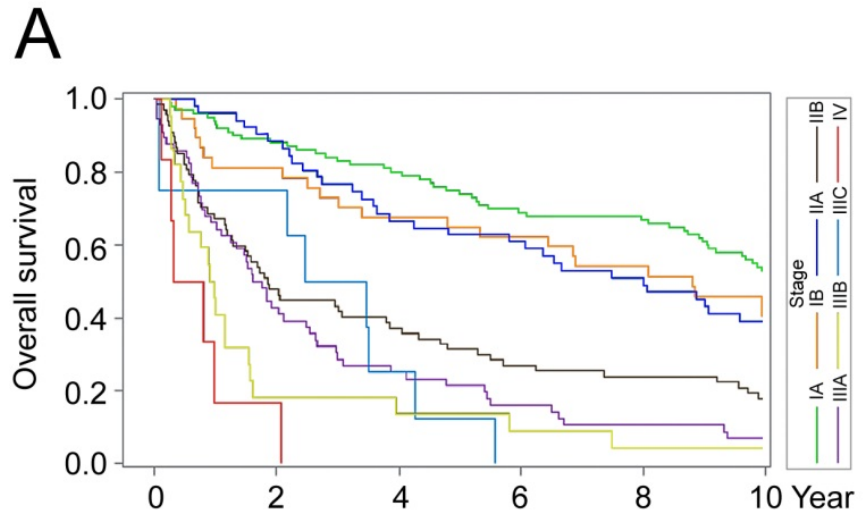

Number of patients remained

$\begin{array}{lcc}\text { IA } & 100 & 88 \\ \text { IB } & 37 & 30 \\ \text { IIA } & 51 & 44 \\ \text { IIB } & 67 & 32 \\ \text { IIIA } & 55 & 24 \\ \text { IIIB } & 22 & 4 \\ \text { IIIC } & 8 & 6 \\ \text { IV } & 6 & 1\end{array}$

$\begin{array}{cc}80 & 69 \\ 25 & 23 \\ 30 & 28 \\ 25 & 18 \\ 14 & 9 \\ 3 & 2 \\ 2 & 0 \\ 0 & 0\end{array}$

Clinicopathological factors associated with the OS in group-3 patients

The demographics and clinicopathological features of group-3 were summarized in Supplementary Table 2. Because TNM staging system could not clearly stratify this group of patients, we analyzed the association between clinicopathological factors and OS. Univariate analysis identified tumor size, age, $\mathrm{N}$ status, $\mathrm{M}$ status, lymphatic invasion, perineural invasion, and GALNT14-rs9679162 genotype $(\mathrm{P}=0.0141)$ as predictors (see Table 2 for all $\mathrm{P}$ values). Stepwise multivariate analysis showed that tumor size $(\mathrm{P}=$ $0.009)$, $N$ status $(P<0.0001), M$ status $(P=0.0096)$, perineural invasion $(\mathrm{P}=0.037)$, and GALNT14-rs9679162 genotype $(\mathrm{P}=0.0482)$ remained in the equation.

Because in group-3 patients, postoperative recurrence occurred more frequently than that in group-1 or -2 patients, we also analyzed the association between clinicopathological factors and time-to-tumor progression (TTP) (Supplementary Table 3). Univariate analysis identified tumor size, age, $\mathrm{T}$ status, $\mathrm{N}$ status, $\mathrm{M}$ status, lymphatic invasion, perineural invasion and GALNT14-rs9679162 genotype $(\mathrm{P}=0.0193)$ as predictors. Stepwise multivariate analysis showed that tumor size $(\mathrm{P}=$ 0.017), $\mathrm{N}$ status $(\mathrm{P}<0.0001), \mathrm{M}$ status $(\mathrm{P}=0.0017)$, perineural invasion $(\mathrm{P}=0.0590)$, and GALNT14-rs9679162 genotype $(\mathrm{P}=0.0710)$ remained in the equation.

B

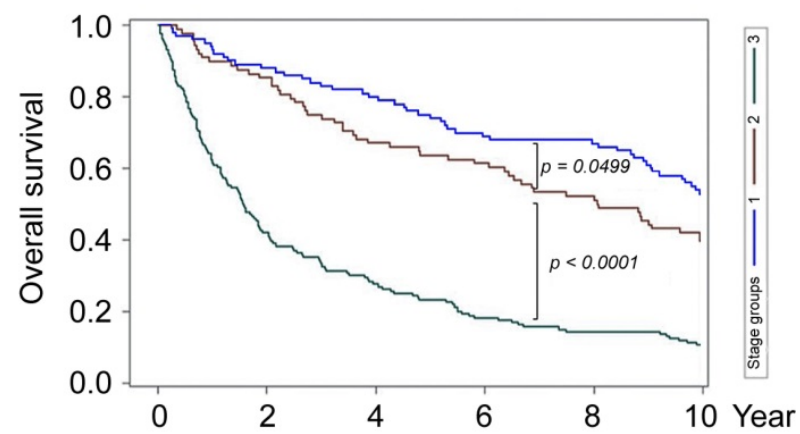

Number of patients remained

$\begin{array}{lcccccc}\text { Group 1 } & 100 & 88 & 80 & 69 & 67 & 53 \\ \text { Group 2 } & 88 & 74 & 55 & 51 & 42 & 32 \\ \text { Group 3 } & 159 & 67 & 44 & 29 & 23 & 17\end{array}$

Figure 1. Kaplan-Meier analysis of postoperative OS in patients with gastric SRCC stratified by tumor stages. (A) Postoperative OS in gastric SRCC patients stratified by the TNM tumor staging method. Different stages were represented by different colors of curves, shown at the right side of the figure. (B) Postoperative OS in gastric SRCC patients stratified by re-classifying the patients into group-1 (stage IA; blue), group-2 (stage IB/IIA; brown), and group-3 (stages IIB through IV; green). 
Table 1. Cox proportional hazard analysis for OS in relation to clinical parameters

\begin{tabular}{|c|c|c|c|c|c|c|c|c|}
\hline \multirow{4}{*}{$\begin{array}{l}\text { Variables } \\
\text { Size }\end{array}$} & & \multicolumn{7}{|c|}{ Cox proportional hazard analysis } \\
\hline & \multicolumn{4}{|c|}{ Univariate } & \multicolumn{4}{|c|}{ Multivariate } \\
\hline & \multirow{2}{*}{$\frac{\mathrm{HR}}{1.104}$} & \multicolumn{2}{|c|}{$95 \% \mathrm{CI}$} & \multirow{2}{*}{$\frac{\mathrm{P}}{<0.0001}$} & \multirow{2}{*}{$\begin{array}{c}\mathrm{HR} \\
1.061\end{array}$} & \multicolumn{2}{|c|}{$95 \% \mathrm{CI}$} & \multirow{2}{*}{$\frac{P}{0.001}$} \\
\hline & & 1.077 & 1.131 & & & 1.024 & 1.1 & \\
\hline Age & 1.02 & 1.011 & 1.028 & $<0.0001$ & & & & \\
\hline Sex & 1.025 & 0.83 & 1.265 & 0.8207 & & & & \\
\hline T status & 1.451 & 1.301 & 1.618 & $<0.0001$ & & & & \\
\hline $\mathrm{N}$ status & 1.997 & 1.749 & 2.281 & $<0.0001$ & 1.749 & 1.346 & 2.272 & $<.0001$ \\
\hline M status & 6.303 & 2.759 & 14.4 & $<0.0001$ & 3.447 & 1.357 & 8.759 & 0.0093 \\
\hline Lymphatic invasion & 1.971 & 1.576 & 2.465 & $<0.0001$ & & & & \\
\hline Vascular invasion & 1.762 & 1.249 & 2.486 & 0.0013 & & & & \\
\hline Perineural invasion & 1.98 & 1.584 & 2.476 & $<0.0001$ & 1.585 & 1.036 & 2.425 & 0.0339 \\
\hline TNM stage $^{\mathrm{a}}$ & 1.705 & 1.491 & 1.949 & $<0.0001$ & & & & \\
\hline GALNT14 genotype ${ }^{\mathrm{b}}$ & 1.179 & 0.919 & 1.512 & 0.1942 & & & & \\
\hline
\end{tabular}

$\mathrm{HR}$, hazard ratio; $\mathrm{CI}$, confidence interval

aTumor stage: divided into IA (group-1), IB+IIA (group-2), and others (group-3)

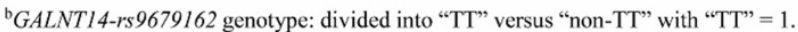

Table 2. Cox proportional hazard analysis for OS in relation to clinical parameters in group-3 patients

\begin{tabular}{|c|c|c|c|c|c|c|c|c|}
\hline \multirow{4}{*}{$\begin{array}{l}\text { Variables } \\
\text { Size }\end{array}$} & \multicolumn{8}{|c|}{ Cox proportional hazard analysis } \\
\hline & \multicolumn{4}{|c|}{ Univariate } & \multicolumn{4}{|c|}{ Multivariate } \\
\hline & \multirow{2}{*}{$\frac{\mathrm{HR}}{1.087}$} & \multicolumn{2}{|c|}{$95 \% \mathrm{CI}$} & \multirow{2}{*}{$\frac{P}{<0.0001}$} & \multirow{2}{*}{$\frac{\mathrm{HR}}{1.062}$} & \multicolumn{2}{|c|}{$95 \% \mathrm{CI}$} & \multirow{2}{*}{$\frac{P}{0.0009}$} \\
\hline & & 1.058 & 1.118 & & & 1.025 & 1.1 & \\
\hline Age & 1.024 & 1.011 & 1.036 & 0.0002 & & & & \\
\hline Sex & 1.154 & 0.844 & 1.576 & 0.3695 & & & & \\
\hline T status & 1.251 & 0.895 & 1.748 & 0.1894 & & & & \\
\hline $\mathrm{N}$ status & 1.491 & 1.177 & 1.890 & 0.0010 & 1.763 & 1.36 & 2.286 & $<0.0001$ \\
\hline M status & 3.064 & 1.334 & 7.037 & 0.0083 & 3.425 & 1.349 & 8.692 & 0.0096 \\
\hline Lymphatic invasion & 1.475 & 1.001 & 2.172 & 0.0002 & & & & \\
\hline Vascular invasion & 1.445 & 0.977 & 2.137 & 0.0654 & & & & \\
\hline Perineural invasion & 1.587 & 1.075 & 2.343 & 0.0201 & 1.562 & 1.027 & 2.376 & 0.037 \\
\hline GALNT14 genotype $\mathrm{a}^{\mathrm{a}}$ & 1.582 & 1.097 & 2.282 & 0.0141 & 0.645 & 1.003 & 2.397 & 0.0482 \\
\hline
\end{tabular}

HR, hazard ratio; CI, confidence interval; "GALNT14-rs9679162 genotype: divided into "TT" versus "non-TT", with "TT" = 1.

A prognosis scoring system to stratify group-3 patients into different prognostic subgroups

By use of the independent predictors identified (Table 2), we took a further step to establish a scoring system to stratify group-3 patients into different prognostic subgroups. Each of the unfavorable predictors was assigned as 1 point when present. As such, it was found that patients could be divided into 3 prognostic subgroups: score $=0 / 1$ (present with equal to or less than 1 unfavorable predictors); score = 2/3 (present with equal to or less than 3 unfavorable predictors); and score $>3$ (present with more than 3 unfavorable predictors) (Fig. 2A). The $\mathrm{P}$ values between these group were all $<0.0001$. The plot overlay these three groups with the IA (group-1) and IB/IIA (group-2) curves were shown as Supplementary Fig. 1. It was found that the best prognostic subgroup from group-3 patients (red line) had OS compatible with that in groups-1 and group-2 patients (stages I to IIA). In addition, the same scoring system could be applied to all SRCC patients for effective prognostic stratification (Fig. 2B). 
A

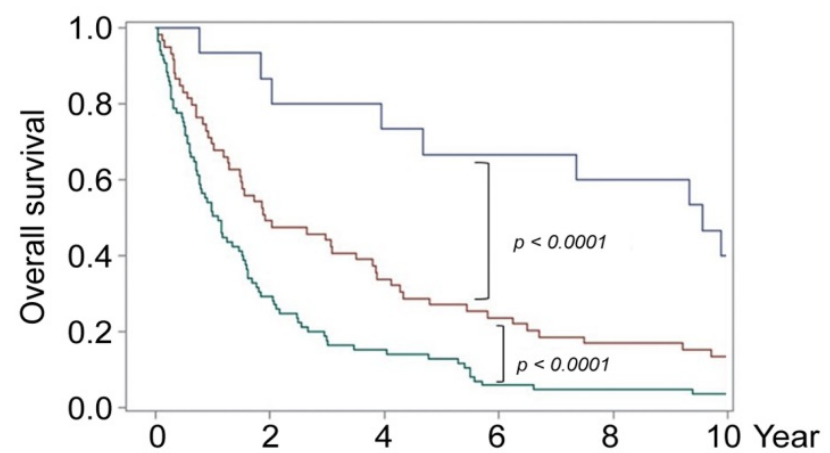

Number of patients remained

$\begin{array}{ccccccc}\text { Score 0/1 } & 15 & 13 & 11 & 10 & 9 & 6 \\ \text { Score 2/3 } & 59 & 29 & 20 & 14 & 10 & 8 \\ \text { Score }>3 & 85 & 25 & 13 & 5 & 4 & 3\end{array}$

B

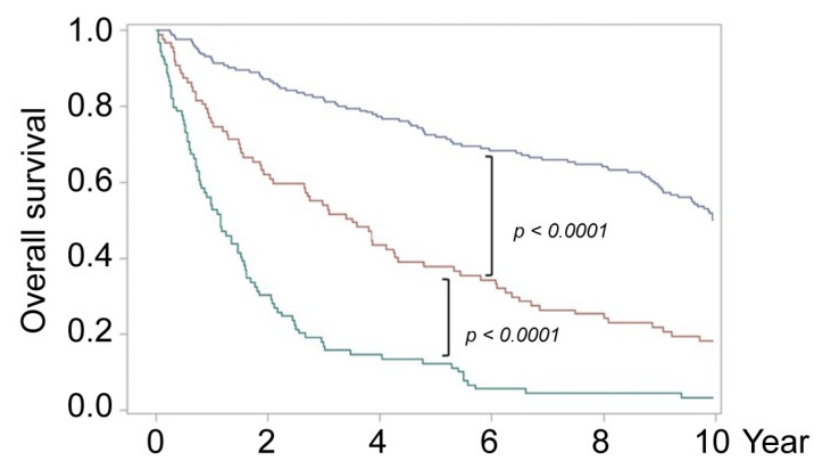

Number of patients remained

$\begin{array}{ccccccc}\text { Score 0/1 } & 155 & 136 & 121 & 107 & 100 & 79 \\ \text { Score 2/3 } & 105 & 70 & 49 & 40 & 31 & 23 \\ \text { Score }>3 & 87 & 24 & 13 & 5 & 4 & 3\end{array}$

Figure 2. Kaplan-Meier analysis of postoperative OS in patients with gastric SRCC stratified by prognosis-related scores. Patients were divided into 3 subgroups with score = 0/1 (grey-blue), 2/3 (red), and >3 (green). (A) All gastric SRCC patients. (B) Advanced (Group-3) SRCC patients.

The disease-free survival of all patients and groups-3 patients could also be divided into 3 prognostic subgroups using this scoring system (Supplementary Fig. 2).

\section{GALNT14-rs9679162 genotype was an effective prognosis predictor in gastric SRCC with more aggressive histological characteristics}

From the Cox proportional hazard analysis, it was found that when all patients were included for analysis, GALNT14-rs9679162 genotype was not associated with OS (Table 1), whereas it became a significant predictor when only group-3 patients were included for analysis (Table $\mathbf{2}$ and Supplementary Table 3). Previous studies in other gastrointestinal cancers showed that GALNT14-rs9679162 genotype was a good predictor in advanced stage colorectal cancer, esophageal cancer, and hepatocellular carcinoma. Therefore, it was possible that the prediction efficacy of GALNT14-rs9679162 genotype was better in advanced rather than early stage cancers. To investigate this hypothesis, we performed a subgroup analysis using forest plot analysis (Fig. 3). It was found that GALNT14-rs9679162 genotype significantly associated with $O S$ in the following subgroups: $\mathrm{N}>0(\mathrm{P}=0.0013)$, lymphatic invasion $(\mathrm{P}=$ $0.021)$, vascular invasion $(P=0.0076)$, perineural invasion $(\mathrm{P}=0.0161)$ and $\mathrm{TNM}$ stage $>$ IIA $(\mathrm{P}<$ 0.0001). In all subgroups, GALNT14-rs9679162 "TT" genotype was associated with a poorer prognosis.

Similarly, when TTP was evaluated, it was found that that GALNT14-rs9679162 genotype significantly associated with TTP in the following subgroups: $\mathrm{N}>0$ $(P=0.0014)$, lymphatic invasion $(P=0.0213)$, vascular invasion $(\mathrm{P}=0.007)$, perineural invasion $(\mathrm{P}=0.0231)$ and TNM stage $>$ IIA $(P=0.0193)$.

\section{Discussion}

The prevalence of gastric SRCC is 16.8-35.5\% among gastric cancer patients [19-21] and the incidence is increasing in recent decade [2]. Although it has been established that gastric cancer with pathological characteristics of signet ring cells harbors a significantly poorer outcome when compared with other types of gastric cancer $[4,22,23]$, the prognostic factors within gastric SRCCs themselves remain poorly defined. In the present study, it was found that a large proportion of gastric SRCC patients were diagnosed in early stages, with $39.48 \%$ in stage I and $14.70 \%$ in stage IIA (Supplementary Table 1). These patients still enjoyed favorable postoperative prognosis (Fig. 1). This finding is in consistent with many previous reports, showing that prognosis of early gastric SRCC is equivalent to or better than that of other GC [23-25]. However, there were still nearly half of our gastric SRCC patients (45.82\%), who had been diagnosed in a more advanced stage at baseline (group-3 patients). In the present study, it was found that the TNM staging method failed to separate these patients into prognosis-distinguishable groups. As the prevalence of gastric SRCC is increasing, it is mandatory to establish an effective prognosis stratification system for these patients. Additionally, a thorough understanding of gastric SRCC prognostic factors could also optimize treatment strategies in the upcoming era of precision medicine. For example, adjuvant targeted therapies could be given to improve survival in SRCC patients who were predicted to have unfavorable postoperative prognosis. 
A

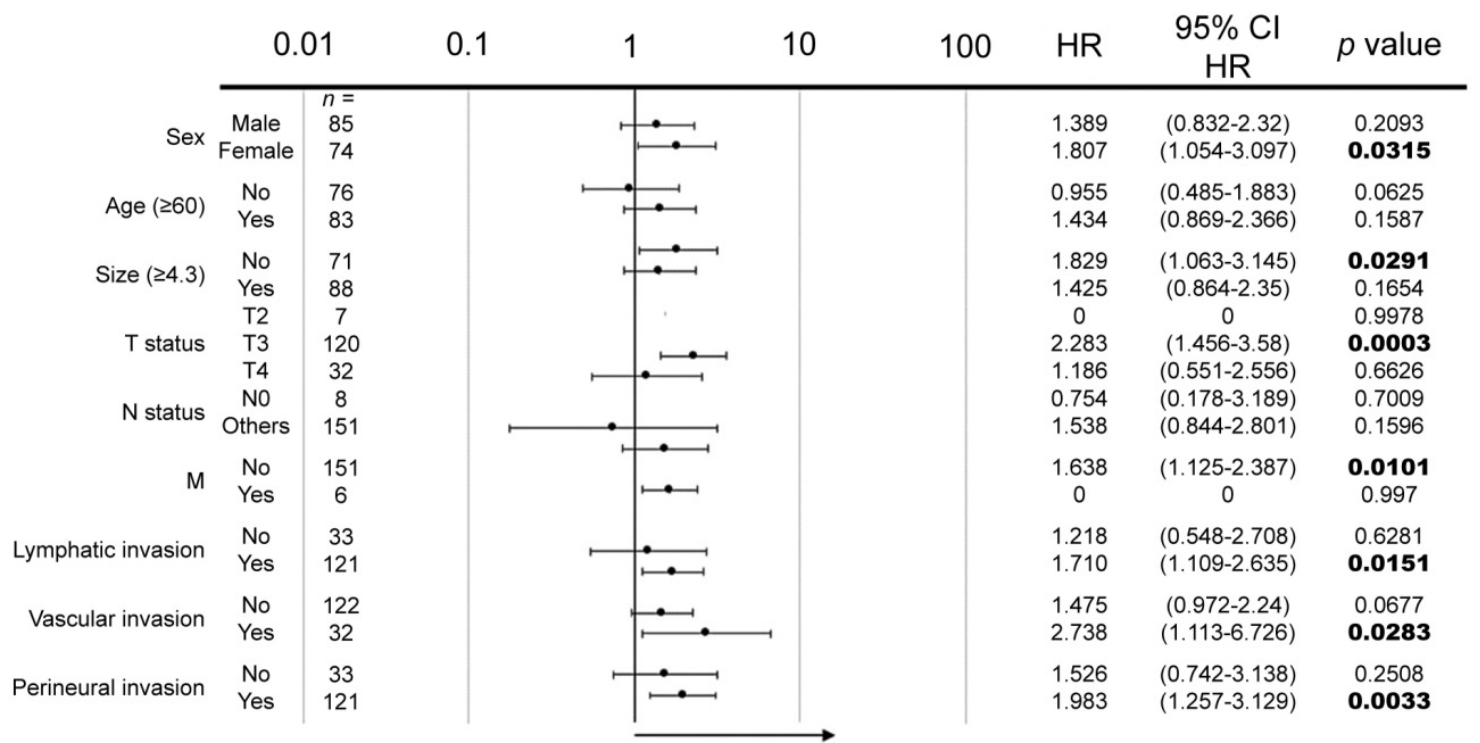

B

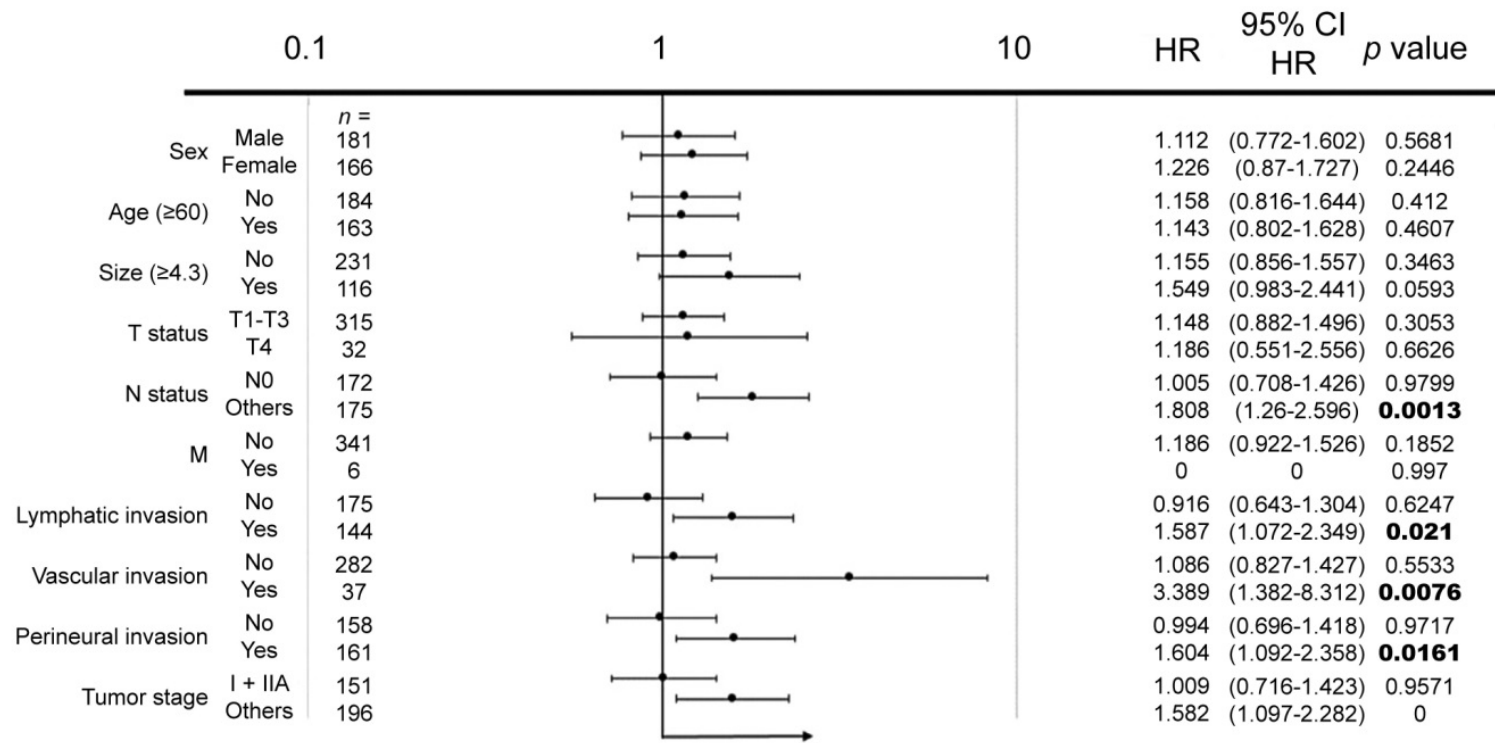

Figure 3. Forest plot subgroup analysis for gastric SRCC patients divided by various clinicopathological characteristics to estimate associations between GALNTI4-rs 9679162 genotype and prognosis. (A) Advanced gastric SRCC (group-3) patients. (B) All gastric SRCC patients included.

At this time, there is no consistent clinicopathological predictors for advanced gastric SRCC patients, let alone molecular biomarkers. Searching through PubMed, it was found that all previous reports had studied whether the pathological characteristic of "signet ring cell" served as a prognostic factor for unfavorable outcomes among all different types of gastric cancers. Only two reports attempted to stratify prognosis within the disease entity of "gastric SRCC". One used lymph-node metastasis system to stratify "early gastric SRCC" [26] and the other showed M2 isoform of pyruvate kinase served as a prognosis factor for gastric SRCC [27]. As such, to the best of our knowledge, our study is the first report combining molecular marker and clinical parameters to predict patients' survivals in "advance stage" gastric SRCC. In the present study, we focused on group-3 patients (more advanced stage) for GALNT14 genotype and clinicopathological factors analysis. In clinicopathological factors analysis, under Cox proportional hazard multivariate models, patients with large tumor size, lymph node metastasis, distant metastasis and perineural invasion had poorer OS, which was in consistent with previously studies [22, 28, 29]. Additionally, we discovered that GALNT14-rs9679162"TT" genotype was also associated with poorer OS in group-3 patients. 
As reported by previous studies, cancer subtypes containing mucin such as mucinous breast and colon cancers were related to unfavorable disease prognosis $[9,30,31]$. However, only a few relevant studies investigated mucinous gastric cancer including gastric SRCC in biomolecular levels. One study proposed that phosphatidylinositol 3-kinase pathway was involved in SRCC carcinogenesis, which bound to ErbB2/ErbB3 complex to activate downstream pathways including p38 MAP kinase, leading to loss of cell-cell contact by crumbling adherent junctions [32]. ErbB2/ErbB3 complex could be activated by MUC4, which belonged to the family of mucins and was overexpressed together with MUC2 in gastric cancer. In SRCC, excessive accumulation of mucins could play a role in carcinogenesis. However, the mechanisms and pathways regarding mucin accumulation and secretion remained poorly understood [33]. On the other hand, $C D H 1$ germline mutations have been recognized as the major cause of hereditary, early-onset, diffuse type gastric cancer. A higher $\mathrm{CDH1}$ mutation detection rate was reported in patients with family history of gastric SRCC and a guideline had been established to recommend genetic testing. E-cadherin is encoded by the CDH1 gene, which is an adhesion molecule highly implicated in oncogenic processes, such as epithelial-mesenchymal transition.

Despite the aforementioned knowledge for oncogenic mechanisms in gastric SRCC, genetic or molecular factors associated with prognosis of SRCC remained poorly studied. GALNT14 encodes an enzyme responsible for the initial step of O-glycosylation in mucin, a heavily O-glycosylated protein in gastrointestinal tract. In hepatocellular carcinoma, GALNT14-rs9679162 was associated with GALNT14 enzyme expression levels in hepatocytes [34]. It is possible that in gastric SRCC, the GALNT14 genotype is also associated with the GALNT14 enzyme expression levels, thereby affecting the O-glycosylation status on mucins. GALNT14-rs9679162 "TT" genotype was associated with poorer outcome in mucinous type stage III colorectal cancer [11]. We hypothesized that it might also play a prognostic role in gastric SRCC, wherein abundant intracellular mucin was present. Our data have clearly confirmed this point. In fact, in subgroups of gastric SRCC patients carrying more aggressive phenotypes such as perineural invasion, lymphatic invasion, and vascular invasion, GALNT14-rs9679162 "TT" genotype could serve as an effective prognosis predictor.

Accordingly, we formulated a prognostic scoring system, including significant prognostic factors (Fig. 2). The scoring system identified a postoperative subgroup with the worse prognosis (green curve) and a subgroup with the most favorable prognosis (blue curve). The outcome of the latter group was compatible with that of early SRCCs (Supplementary Fig. 1). Such a scoring system could be applied to precision medicine practicing. For example, in the future, SRCCs with the worse prognosis could be further provided with adjuvant therapy, such as TS-1, following surgical resection.

\section{Conclusion}

Patients with GALNT14-rs9679162 "TT" genotype predicted unfavorable postoperative prognosis in advanced gastric SRCC. By combining GALNT14 genotype and clinicopathological factors, advanced SRCCs could be further stratified into prognosis distinguishable subgroups for treatment optimization.

\section{Supplementary Material}

Supplementary figures and tables. http://www.jcancer.org/v09p3540s1.pdf

\section{Acknowledgements}

The authors thank all the members of the Cancer Center, Chang Gung Memorial Hospital, for their invaluable help. This study was supported by Chang Gung Medical Research Program (CMRPG3F1602).

\section{Ethical Standards}

All procedures followed were in accordance with the ethical standards of the responsible committee on human experimentation (institutional and national) and with the Helsinki Declaration of 1964 and later versions. The study protocol was approved by the Institutional Review Board of Chang Gung Memorial Hospital (104-8623B). Written informed consent was obtained for all participants, before surgical samples were deposited to Tissue Bank of Chang Gung Memorial Hospital.

\section{Competing Interests}

The authors have declared that no competing interest exists.

\section{References}

1. Ferlay J, Soerjomataram I, Dikshit R, et al. Cancer incidence and mortality worldwide: sources, methods and major patterns in GLOBOCAN 2012. Int J Cancer. 2015; 136: E359-86.

2. Henson DE, Dittus C, Younes M, et al. Differential trends in the intestinal and diffuse types of gastric carcinoma in the United States, 1973-2000: increase in the signet ring cell type. Arch Pathol Lab Med. 2004; 128: 765-70.

3. Hyung WJ, Noh SH, Lee JH, et al. Early gastric carcinoma with signet ring cell histology. Cancer. 2002; 94: 78-83.

4. Kim JP, Kim SC, Yang HK. Prognostic significance of signet ring cell carcinoma of the stomach. Surg Oncol. 1994; 3: 221-7.

5. Lauwers G CF, Graham D, Curado M, et al. Classification of Tumours of the Digestive System, 4th ed. Lyon: IARC Press; 2010: 48-58. 
6. Piessen G, Messager M, Leteurtre E, et al. Signet ring cell histology is an independent predictor of poor prognosis in gastric adenocarcinoma regardless of tumoral clinical presentation. Ann Surg. 2009; 250: 878-87.

7. Ribeiro MM, Sarmento JA, Sobrinho Simoes MA, et al. Prognostic significance of Lauren and Ming classifications and other pathologic parameters in gastric carcinoma. Cancer. 1981; 47: 780-4.

8. Taghavi S, Jayarajan SN, Davey A, et al. Prognostic significance of signet ring gastric cancer. J Clin Oncol. 2012; 30: 3493-8.

9. Theuer $C P$, Nastanski F, Brewster WR, et al. Signet ring cell histology is associated with unique clinical features but does not affect gastric cancer survival. Am Surg. 1999; 65: 915-21.

10. Shamseldin HE, Tulbah M, Kurdi W, et al. Identification of embryonic lethal genes in humans by autozygosity mapping and exome sequencing in consanguineous families. Genome Biol. 2015; 16: 116.

11. De Mariano M, Gallesio R, Chierici M, et al. Identification of GALNT14 as a novel neuroblastoma predisposition gene. Oncotarget. 2015; 6: 26335-46.

12. Liang $\mathrm{KH}, \mathrm{Yeh} \mathrm{TS}, \mathrm{Wu} \mathrm{RC}$, et al. GALNT14 genotype is associated with perineural invasion, lymph node metastasis and overall survival in resected cholangiocarcinoma. Oncol Lett. 2017; 13: 4215-23.

13. Lin WR, Chiang JM, Liang KH, et al. GALNT14 Genotype Predicts Postoperative Outcome of Stage III Colorectal Cancer With Oxaliplatin as Adjuvant Chemotherapy. Medicine (Baltimore). 2016; 95: e3487.

14. Lin WR, Hsu CW, Chen YC, et al. GALNT14 genotype, alpha-fetoprotein and therapeutic side effects predict post-chemotherapy survival in patients with advanced hepatocellular carcinoma. Mol Clin Oncol. 2014; 2: 630-40.

15. Tsou YK, Liang KH, Lin WR, et al. GALNT14 genotype as a response predictor for concurrent chemoradiotherapy in advanced esophageal squamous cell carcinoma. Oncotarget. 2017; 8: 29151-60.

16. Edge SB BD, Compton CC, Fritz AG, et al. American Joint Committee on Cancer: AJCC cancer staging manual, 7th ed. New York: Springer; 2009.

17. Liang KH, Yang PC, Yeh CT. Genotyping the GALNT14 gene by joint analysis of two linked single nucleotide polymorphisms using liver tissues for clinical and geographical comparisons. Oncol Lett. 2014; 8: 2215-20.

18. Yeh $\mathrm{CT}$, Liang $\mathrm{KH}$, Lin $\mathrm{CC}$, et al. A single nucleotide polymorphism on the GALNT14 gene as an effective predictor of response to chemotherapy in advanced hepatocellular carcinoma. Int J Cancer. 2014; 134: 1214-24.

19. Huh CW, Jung DH, Kim JH, et al. Signet ring cell mixed histology may show more aggressive behavior than other histologies in early gastric cancer. J Surg Oncol. 2013; 107: 124-9.

20. Kim DY, Park YK, Joo JK, et al. Clinicopathological characteristics of signet ring cell carcinoma of the stomach. ANZ J Surg. 2004; 74: 1060-4

21. Zhang $\mathrm{M}$, Zhu $\mathrm{G}$, Zhang $\mathrm{H}$, et al. Clinicopathologic features of gastric carcinoma with signet ring cell histology. J Gastrointest Surg. 2010; 14: 601-6.

22. Bozkaya Y, Erdem GU, Ozdemir NY, et al. Comparison of clinicopathological and prognostic characteristics in patients with mucinous carcinoma and signet ring cell carcinoma of the stomach. Curr Med Res Opin. 2017; 33: 109-16.

23. Jiang CG, Wang ZN, Sun Z, et al. Clinicopathologic characteristics and prognosis of signet ring cell carcinoma of the stomach: results from a Chinese mono-institutional study. J Surg Oncol. 2011; 103: 700-3.

24. Ha TK, An JY, Youn HK, et al. Indication for endoscopic mucosal resection in early signet ring cell gastric cancer. Ann Surg Oncol. 2008; 15: 508-13.

25. Kunisaki C, Shimada $H$, Nomura $M$, et al. Therapeutic strategy for signet ring cell carcinoma of the stomach. Br J Surg. 2004; 91: 1319-24.

26. Pyo JH, Shin $\mathrm{CM}$, Lee $\mathrm{H}$, et al. A risk-prediction model based on lymph-node metastasis for incorporation into a treatment algorithm for signet ring cell-type intramucosal gastric cancer. Ann Surg. 2016;264:1038-1043.

27. Lim JY, Yoon SO, Seol SY, et al. Overexpression of the M2 isoform of pyruvate kinase is an adverse prognostic factor for signet ring cell gastric cancer. World J Gastroenterol. 2012;18:4037-43.

28. Kong P, Wu R, Yang C, et al. Prognostic Impact of the Signet Ring Cell Type in Node-Negative Gastric Cancer. Sci Rep. 2016; 6: 26313.

29. Voron T, Messager M, Duhamel A, et al. Is signet-ring cell carcinoma a specific entity among gastric cancers? Gastric Cancer. 2016; 19: 1027-40.

30. Lam WW, Chu WC, Tse GM, et al. Sonographic appearance of mucinous carcinoma of the breast. AJR Am J Roentgenol. 2004; 182: 1069-74.

31. Wu C, Guo X, Wang W, et al. N-Acetylgalactosaminyltransferase-14 as a potential biomarker for breast cancer by immunohistochemistry. BMC Cancer. 2010; 10: 123.

32. Fukui Y. Mechanisms behind signet ring cell carcinoma formation. Biochem Biophys Res Commun. 2014; 450: 1231-3.

33. Pernot S, Voron T, Perkins G, et al. Signet-ring cell carcinoma of the stomach: Impact on prognosis and specific therapeutic challenge. World J Gastroenterol. 2015; 21: 11428-38.

34. Tran DT, Ten Hagen KG. Mucin-type O-glycosylation during development. J Biol Chem. 2013; 288: 6921-9. 